\title{
A study to assess the knowledge and awareness related to radiographic protection in undergraduate dental students
}

\author{
Jasdeep Kaur Cheema ${ }^{1}$, Lakshay Dhawan ${ }^{2}$, Geetanjali ${ }^{3}$, Jaswant Singh Toor ${ }^{4}$, Harkanwal Preet Singh ${ }^{5, *}$ \\ ${ }^{1,3}$ Private Practitioner, ${ }^{2}$ Clinical Associate, ${ }^{4}$ Lecturer, ${ }^{5}$ Associate Professor, ${ }^{\mathbf{A}}$ Amritsar, Punjab, ${ }^{2}$ Interventurus Knowledge Solutions, Navi \\ Mumbai, Maharashtra, ${ }^{3}$ Dento-Med Health Care, Amritsar, Punjab, ${ }^{4}$ Dept. of Pedodontics, ${ }^{5}$ Dept. of Oral Pathology, ${ }^{4,5}$ Dasmesh Institute \\ of Research and Dental Sciences, Faridkot, Punjab, India
}

*Corresponding Author: Harkanwal Preet Singh

Email: hkps0320@gmail.com

\begin{abstract}
Introduction: In the field of dentistry, X-rays are utilized broadly for diagnosing dental diseases as well as providing therapeutic means. ${ }^{1}$ $\mathrm{X}$-rays are used from mild conditions such as incipient caries to severe conditions such as alveolar bone loss and implant placement. Hence, dental therapist, office staff and the patients are at high risk to exposure of ionizing radiations.

Aim of the Study: To study the knowledge and assessment related to radiographic protection in undergraduate dental students.

Materials and Methods: The study was conducted among undergraduate dental students. For the study, we selected undergraduate dental students from 3rd year, 4th year and internship. A total of 120 students were included in the study. A questionnaire related to radiation protocol in the form of multiple choices was given to each participant and the response sheets were collected after 30 minutes.

Results: A total of 120 undergraduate students were included in the study. The number of students from third year were 50, from final year were 39 and from internship were 31 . We observed that in $3^{\text {rd }}$ year, students with low knowledge were 24 , moderate knowledge were 12 and with sufficient knowledge were 14 . In $4^{\text {th }}$ year, students with low knowledge were 12 , moderate knowledge were 15 and with sufficient knowledge were 12 .

Conclusion: The interns and final year students are more knowledgeable and aware of radioprotection and its harmful effects on exposure as compared to third year students. The rigorous clinical training along with extensive theoretical teaching provided to final year students and interns posted in clinical departments, likely to be responsible for this difference in knowledge of students.
\end{abstract}

Keywords: Dental students, Radiographic protection, X-rays.

\section{Introduction}

In the field of dentistry, X-rays are utilized broadly for diagnosing dental diseases as well as providing therapeutic means. ${ }^{1} \mathrm{X}$-rays are used from mild conditions such as incipient caries to severe conditions such as alveolar bone loss and implant placement. The most commonly used techniques which involve X-rays range from intraoral radiographs to cone beam computed tomography. ${ }^{2}$ Hence, dental therapist, office staff and the patients are at high risk to exposure of ionizing radiations. Hence, knowledge of protection from these harmful radiations which are of significant importance to dentistry is required by every dentist to protect himself and others in the dental office. ${ }^{3,4} \mathrm{~A}$ person exposed to harmful ionizing radiations is at high risk for adverse effects on hematopoietic, immune, reproductive, circulatory, respiratory, musculoskeletal, endocrine, nervous, digestive, and urinary systems. Some other harmful and deliberating effects of radiations include skin burns, leukemia, and other types of cancers. ${ }^{5}$ Studies have shown that long-term radiation exposure as well as long-term low dose exposure to ionizing radiation tend to induces risks such as mutagenic and carcinogenic hazards. In the dental colleges, the dental students posted in the clinical departments are high risk to radiation exposure due to lack of proper knowledge and training of radiation exposure. ${ }^{6}$ Hence, we planned the to study the knowledge and assessment related to radiographic protection in undergraduate dental students.

\section{Materials and Methods}

The study was conducted among undergraduate dental students. For the study, we selected undergraduate dental students from 3rd year, 4th year and internship. A total of 120 students were included in the study. A questionnaire related to radiation protocol in the form of multiple choices was given to each participant and the response sheets were collected after 30 minutes. The questionnaire was a selfprepared, pilot-tested and it was specially designed for the study. An informed verbal consent was obtained from each subject. The questionnaires were marked and the participants were grouped according to the scores. Participants with score 0-6 were grouped as low knowledge group, with score 7-12 were grouped as moderate knowledge and with score 13-18 were grouped as sufficient knowledge. The data was entered in a excel file and stored for further analysis.

The statistical analysis of the data was done using SPSS version 20.0 for windows. We used Student's t-test for analyzing the results. Statistical significance was predetermined at $\mathrm{p}$-value less than 0.05 .

\section{Results}

A total of 120 undergraduate students were included in the study. Table 1 shows the demographic details of the patients. The number of students from third year were 50, from final year were 39 and from internship were 31. Table 2 shows the scoring of patients based on the knowledge of radiographic protection. We observed that in $3^{\text {rd }}$ year, students with low knowledge were 24, moderate knowledge 
were 12 and with sufficient knowledge were 14. In $4^{\text {th }}$ year, students with low knowledge were 12, moderate knowledge were 15 and with sufficient knowledge were 12. In internship, students with low knowledge were 24 , moderate knowledge were 12 and with sufficient knowledge were 14. (Fig. 1)

Table 1: Demographic details of the patients

\begin{tabular}{|l|c|c|c|}
\hline \multirow{2}{*}{ Academic year } & \multicolumn{2}{|c|}{ Gender } & \multirow{2}{*}{ Total } \\
\cline { 2 - 3 } & Male & Female & \\
\hline $3^{\text {rd }}$ year & 22 & 28 & 50 \\
\hline $4^{\text {th }}$ year & 18 & 21 & 39 \\
\hline Internship & 11 & 20 & 31 \\
\hline
\end{tabular}

Table 2: Scoring of patients based on the knowledge of radiographic protection

\begin{tabular}{|l|c|c|c|}
\hline Academic year & Low knowledge & Moderate knowledge & Sufficient knowledge \\
\hline $3^{\text {rd }}$ year & 24 & 12 & 14 \\
\hline $4^{\text {th }}$ year & 12 & 15 & 12 \\
\hline Internship & 7 & 10 & 14 \\
\hline
\end{tabular}

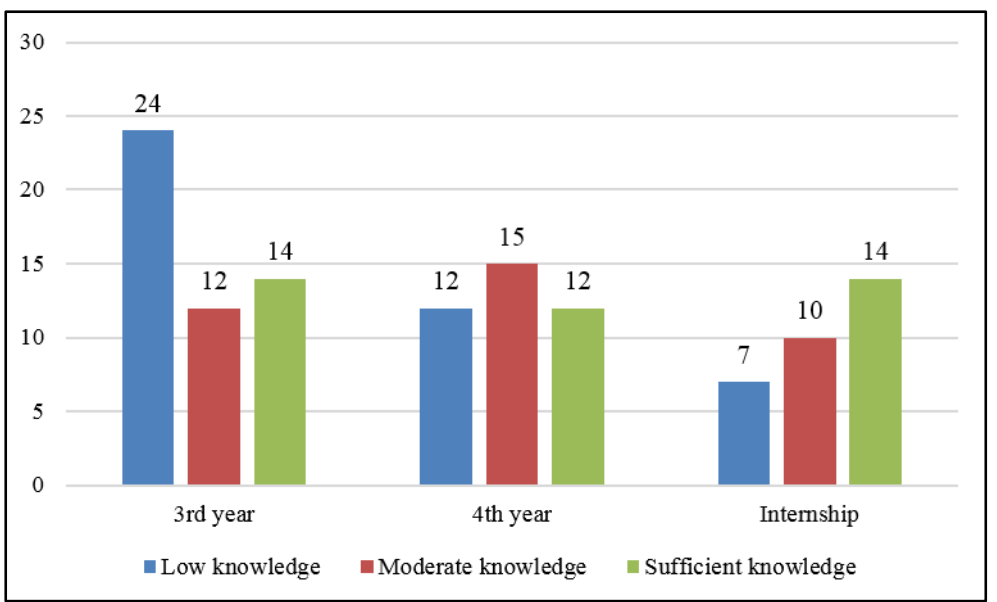

Fig. 1: Scoring of patients based on the knowledge of radiographic protection

\section{Discussion}

In the present study, we studied the knowledge and assessment related to radiographic protection in undergraduate dental students. We observed that majority of students in internship had sufficient knowledge in regards to radiographic protection; however, majority students in $3^{\text {rd }}$ year had low knowledge or moderate radiographic protection knowledge. The results were statistically nonsignificant. The results were compared with previous studies and results were consistent with previous studies. Dianati M et al performed a study on ICU nurses. They were evaluated for radiation protection knowledge by using a questionnaire. They had selected a total of 44 nurses posted in intensive care units. From the results of the study, it was observed that the mean score for knowledge of radiation protection in nurses was $4.77 \pm 1.38$ it was recommended that regular educative programs on radiation protection should be organized to educate the hospital staff including nurses. Binnal A et al studied a subpopulation of Indian dentist to analyse the knowledge of radiation protection among them using a pre-tested questionnaire. Collection of demographic data such as age, gender, education level and duration of practice was done. A total of 87 practitioners participated in the study. The mean score for knowledge on radiation protection among study population was 59.39 \pm 7.01 , however, the mean practice score of the dentists was $5.80 \pm 3.19$. They concluded that private dentists do not have sufficient radioprotection knowledge. ${ }^{7,8}$

Kada $S$ et al performed a study on the final year students of MBBS by sending them online questionnaires to check the knowledge of radiation dosage and harmful effects of radiation exposure. The questionnaire consisted of multiple choice questions on radiation protection and risks to radiation exposure. Correct answer was given one mark no mark was given for incorrect answer. The highest score a person can score was 11 and lowest was 0 . The competed questionnaire was received from 99 students. They observed that the mean score for radiation protection knowledge of medical students was 3.91 out of 11 . More than 5 scores were scored by only $50 \%$ students.it was concluded that knowledge of radiation dosage and harmful effects of radiation exposure was low. Chaudhry $\mathrm{M}$ et al conducted a questionnaire based study among general dentists in the National Capital Region for knowledge on radiation safety during routine dental practices involving $\mathrm{X}$-rays. The questionnaire was sent to a total of 500 dentists in NCR 
region. Out of 500 dentists whom we had sent the questionnaire, $70.6 \%$ responded to our questionnaire. They observed that $64.8 \%$ dentists regarded thyroid to be the most important vital organ for radiation safety. Also, position and distance rule were followed by just $28.8 \%$ of dentists. They concluded that knowledge and behavior of radiation safety among the dentists in NCR is not satisfactory and strict rules should be implemented with regular dental programs on educating radiation protection to practicing dentists should be conducted by the state regularly. ${ }^{9,10}$

\section{Conclusion}

Within the limitations of the study we conclude that interns and final year students are more knowledgeable and aware of radioprotection and its harmful effects on exposure as compared to third year students. The rigorous clinical training along with extensive theoretical teaching provided to final year students and interns posted in clinical departments, likely to be responsible for this difference in knowledge of students.

\section{Conflict of Interest: Nil.}

\section{References}

1. Briggs-Kamara MA, Okoye PC, Omubo-Pepple VB. Radiation safety awareness among patients and radiographers in three hospitals in Port Harcourt. Am J Sci Ind Res. 2013;4:83-88.

2. Langlais RP, Langland OE. Risks from dental radiation in 1995. J Calif Dent Assoc. 1995;23:33.

3. Stewart F, Akleyev A, Hauer-Jensen M, Hendry J, Kleiman N, Macvittie T, et al. ICRP publication 118: ICRP statement on tissue reactions and early and late effects of radiation in normal tissues and organs-threshold doses for tissue reactions in a radiation protection context. Ann ICRP. 2012;41:1-322.

4. Popanda O, Marquardt JU, Chang-Claude J, Schmezer P. Genetic variation in normal tissue toxicity induced by ionizing radiation. Mutat Res. 2009;667:58-69.

5. Brindle MJ, RCR Working Party. Making the best use of a Department of Clinical radiology: guidelines for doctors (4 ed) London: The Royal College of Radiologists; 1998. p. 13.

6. Soye JA, Paterson A. A survey of awareness of radiation dose among health professionals in Northern Ireland. Br J Radiol. 2008;81(969):725-729. doi: 10.1259/bjr/94101717.

7. Dianati M, Zaheri A, Talari HR, Deris F, Rezaei S. Intensive Care Nurses' Knowledge of Radiation Safety and Their Behaviors Towards Portable Radiological Examinations. Nur Midwifery Stud. 2014;3(4):e23354.

8. Binnal A, Rajesh G, Denny C, Ahmed J, Nayak V. Insights into the state of radiation protection among a subpopulation of Indian dental practitioners. Imaging Sci Dent. 2013;43(4):253259.

9. Kada S. Awareness and knowledge of radiation dose and associated risks among final year medical students in Norway. Insights into Imaging. 2017;8(6):599-605.

10. Chaudhry M, Jayaprakash K, Shivalingesh KK. Oral Radiology Safety Standards Adopted by the General Dentists Practicing in National Capital Region (NCR). J Clini Diagn Res.2016;10(1):ZC42-ZC45.

How to cite this article: Cheema J. K, Dhawan L,
Geetanjali, Toor J. S, Singh H. P. A study to assess the
knowledge and awareness related to radiographic
protection in undergraduate dental students. Int J
Maxillofac Imaging. 2018;4(4):131-133.

